\title{
Brain Derived Neurotrophic Factor: From Neurobiology to Sexual Risk Prevention
}

\author{
María José Míguez ${ }^{1 *}$, Luis A Espinoza², Caroline Perez ${ }^{1}$ and Christopher Kahler ${ }^{3}$ \\ ${ }^{1}$ Health Behavior and Policy Initiative, School of Integrated Science and Humanity, Florida International University, Miami, USA \\ ${ }^{2}$ Division of Infectious Diseases, Department of Medicine, University of Miami, Miami, USA \\ ${ }^{3}$ Department of Behavioral and Social Sciences, Brown University School of Public Health, Brown University, Providence, Rhode Island, USA
}

\section{Abstract}

Objective: The authors' draw on neurobiological theory to posit that sexual risks might be associated with neurological changes, specifically, those neuro-adaptive changes associated with addictions. We propose that low levels of brain-derived neurotrophic factor (BDNF), a signaling molecule involved in cognitive, emotional, and addiction behaviors, may play a key role in the expression of high-risk sexual behaviors.

Methods: In a prospective cohort of 400 people living with HIV (PLWH), information was collected regarding smoking (Fagerström Test for Nicotine Dependence), hazardous alcohol use (men $>14$ and women $>7$ drinks/week), sexual behaviors. To assess the role of BDNF, platelet poor plasma was obtained to measure BDNF levels and participants were dichotomized into Groups 1 (BDNF $\leq 5000 \mathrm{pg} / \mathrm{mL}$ ) and 2 (BDNF >5000 pg/mL).

Results: Over two-thirds of the participants reported multiple risk behaviors, confirming our hypothesis that Group 1 would have a general risk-taking tendency. Individuals in Group 1 were more likely to smoke $(p=0.0001)$ and to drink alcohol hazardously (HAU) more days per week $(p=0.04)$, particularly hard liquor $(p=0.001)$. In support of our hypothesis, Group 1 was also more likely to report sex under the influence of drugs/alcohol $(p=0.003)$ and to exchange sex for money $(p=0.03)$. Men from Group 1 had multiple partners $(p=0.09)$, and engaged in unprotected anal sex $(p=0.08)$ more than those in Group 2. The results from regressions showed that BDNF $(p=0.05)$, use of antidepressants $(p=0.003)$, hazardous alcohol use $(p=0.0001)$ and the BDNF $\times$ gender interaction $(p=0.022)$ were significant predictors of high-risk sexual behaviors. Further supporting our postulate, participants with low BDNF showed triple the risk of herpes $(p=0.05)$.

Conclusion: This innovative approach demonstrated for the first time that substance abuse disorders (especially alcohol abuse), high-risk sexual behaviors, and sexually transmitted diseases occur more frequently in individuals with low BDNF levels. These findings suggest the importance of using BDNF modifiers in preventive interventions concerning people living with HIV. Given the limitations of the design, new studies among the general population are needed to confirm the generalizability of the findings.

Keywords: Human immunodeficiency virus (HIV); Hazardous alcohol use (HAU); Sexual risk behaviors; Brain derived neurotrophic factor (BDNF); Binge drinking; People living with HIV (PLWH); Smoking

\section{Introduction}

Although HIV cases have been reported across the nation, the impact of the epidemic is not uniformly distributed. The Southern United States contributes about half of the total reported cases and Miami, ranks second for the number of both total and new HIV cases [1]. Despite strenuous public health efforts, in the past years the annual incidence of HIV has not declined significantly (from 130,000 in the 1980s to 50,000) 1. Accordingly, the new goal of the National HIV/ AIDS Strategy is to decrease the annual number of new infections by $25 \%$ by 2020 [2]. To achieve this milestone, ongoing research is required to determine which factors are associated with HIV-related sexual risk behaviors and can be affected through intervention(s). Research should focus on identifying why some people continue to engage in high-risk sexual practices despite sufficient knowledge of the risks. These efforts should be intensified in geographic areas such as the South, where the HIV epidemic is heavily concentrated. It is also critical to expand the current prevention armamentarium with evidence-based, scalable interventions.

Smoking, drinking, drug use and unprotected sex are some of the many ways individuals engage in risk-taking. Unfortunately, risk behaviors tend to present themselves in clusters [3]. Though scientists have raised the question of whether third variables may account for the co-occurrence of such behaviors, the literature is quite limited in this area. Yet, according to a neurobiological theory risk taking (i.e., drug abuse) is associated with alterations on the cognitive system responsible for controlling impulses, planning ahead, and using executive functions to avoid taking unnecessary risks. This system is related to the development of the prefrontal cortex and the increased connectivity between cortical and subcortical regions [4].

In line with this model, our group has been investigating the involvement of brain-derived neurotrophic factor (BDNF) in risktaking (i.e., hazardous alcohol use and smoking) in PLWH. BDNF was selected because of its critical role in higher-order cognitive functions, neuronal apoptosis, plasticity and mood, all of which control risk-taking [5]. In addition, our group has demonstrated that BDNF

*Corresponding author: Maria Jose Miguez-Burbano, MD, PhD, Florida International University/School of Integrated Sciences and Humanity AHC4 - 355, 11200 S.W. 8th St., Miami, FL 33199; Tel: 561-422-3610; E-mail: mjmiguez@fiu.edu

Received September 08, 2016; Accepted September 26, 2016; Published October 03, 2016

Citation: Míguez MJ, Espinoza LA, Perez C, Kahler C (2016) Brain Derived Neurotrophic Factor: From Neurobiology to Sexual Risk Prevention. J AIDS Clin Res 7: 620. doi: 10.4172/2155-6113.1000620

Copyright: (c) 2016 Míguez MJ, et al. This is an open-access article distributed under the terms of the Creative Commons Attribution License, which permits unrestricted use, distribution, and reproduction in any medium, provided the original author and source are credited. 
homeostasis is directly altered by the human immunodeficiency virus, and is particularly dysfunctional in patients with substance-related addictions, such as hazardous alcohol use and tobacco dependence $[6,7]$. Consistent with these BDNF roles, it is conceivable that a decrease in BDNF signaling could profoundly alter sexual behaviors in PLWH by interfering with cognitive function and impulse control.

Furthermore, BDNF can impact serotonin and dopamine, which studies suggest can control sexual motivation, copulatory behavior and time of ejaculation [8]. In despite of that, neurotrophic factors have been typically neglected in prior decision-making and sexual risk behavior models. Therefore, as a logical advancement of our prior work, these analyses focused on the potential role of BDNF alterations on sexual risk-taking behaviors in PLWH in South Florida, one of the epicenters of the epidemic [9]. If correct, findings can explain the close association between HIV, and alcohol and sexual risk behaviors. Our model can also justify the adoption of generalized risk-taking as a concept and strengthen and expand existing knowledge of HIV risk behaviors.

\section{Methods}

\section{Study population}

The Platelet Alcohol Disorders Study (2010-2013) consists of 400 PLWH who are at least 18 years old and under regular care at Miami's primary open-access public health system. Non-ambulatory patients were excluded, as well as those with major comorbidities, including: central nervous system (CNS) opportunistic infections, head injury with or without loss of consciousness, tumors, major psychiatric disease, developmental disorders, severe malnutrition, or confirmed cardiovascular or immune based disease, (i.e., malignancies/ autoimmune diseases/arthritis). Since the study was focusing on the consequences of hazardous alcohol use, participants who had cirrhosis, active viral hepatitis and dependent drug users were excluded.

The study was designed and conducted according to the principles expressed in the Declaration of Helsinki. The protocol was approved by the Institutional Review Boards at Florida International University and the University of Miami. Those participants who provided written informed consent, and that signed a medical release form, were consequently enrolled and followed over a period of six months.

\section{Participants' assessment protocol}

Upon entry to the study, information was obtained in person using structured questionnaires. Blood was drawn for BDNF, serotonin and immunological evaluation.

\section{Sexual risk and protective behaviors}

The questionnaire began with an introduction-and the definition of sexual terms to minimize ambiguity. A main partner was defined as someone to whom the participant felt most committed (e.g. boyfriend or girlfriend, spouse, or life partner). A casual partner was someone to whom the participant did not feel committed or did not know very well. A client was one with whom the participant had sex in exchange for something, such as money or drugs (i.e., exchange sex). Participants were instructed to think back over the past 6 and 12 months and respond as accurately as possible. A six-month retrospective recall period was selected because previous research has shown more reliable reports for number of partners and sexual events over this time period, compared to more recent behaviors [10]. Separate, but equivalent versions were used for males and females, each with gender appropriate language. The survey was composed of 20 multiple-choice questions regarding sexual behaviors in the past 6 months. Some items required selection of a single choice; others permitted checking all responses that applied. The content domain of survey questions included number of multiple and/or concurrent partners and type. Participants could choose having more than one answer. Information was also collected regarding the sero-status of the sexual partner (sero-concordant, sero-discordant, unknown). The survey included questions about types of sexual intercourse (oral, anal, and vaginal), frequency (always, frequently, rarely, once, never) and condom use (always, frequently, sometimes, never). Condom use inquiries encompassed not only frequency, but type of partner, and condom use during anal, vaginal, or oral sex. Consistent condom use was characterized by an "always" response. The survey also examined alcohol and/or drug use proximal to sexual encounters (i.e., before or during). Based on the literature, the following were rated as high risk sexual practices: unprotected anal intercourse, sex during menses, multiple partners, having sex for money and use of hazardous alcohol/drugs close to intercourse.

\section{Other risky behaviors}

At each visit, PLWH reported alcohol intakes for the past six months using two brief, standardized and validated screening questionnaires: The Alcohol Use Disorders Identification Test (AUDIT) and the Alcohol Dependence Scale (ADS). All the assessments were preceded by the following introduction: "Consider a 'drink' to be a $12 \mathrm{oz}$ can or bottle of beer, a $4 \mathrm{oz}$ glass of wine, a wine cooler, 1 cocktail or a shot (1.25 oz) of hard liquor (like gin or vodka)." Alcohol consumption scores were computed by averaging cross products of quantity and frequency of beer/wine/hard liquor. Based on the National Institute of Alcohol Abuse and Alcoholism criteria, men who reported $>14$ and women $>7$ drinks/week were classified in the hazardous drinkers group, while those who reported fewer drinks were included in the non-hazardous drinkers group. Subjects reporting drinking 5 or more drinks on the same occasion on each of 5 or more days in the past 30 days were classified as heavy drinkers and given 2 points in the risk scale [11].

The Fagerström Test for Nicotine Dependence (FTND 97), a standardized questionnaire extensively used in research, was used to assess smoking habits [12]. The FTND incorporates questions about the age of initiation, number of years smoking, number of cigarettes smoked per day, and signs/symptoms of addiction.

\section{Decision making/impulsivity}

The computerized version of the Wisconsin Card Sorting Task64 (WCST-64) is a widely used test of executive functioning, but particularly inhibition and shifting. In this test subjects are asked to match a virtual card with four cards presented on the computer screen. After the participant makes ten consecutive correct matches, the program switches to matching on a different criterion, without prior notification. Measures of performance include the number of categories completed, total errors and perseverative errors. When a participant who chooses five consecutive correct answers suddenly matches on another criterion, this shift is considered a manifestation of cognitive impulsivity and is called "failure to maintain set." [13].

\section{BDNF}

Circulating levels of BDNF were selected because prior studies have demonstrated that, although different from those in the cerebrospinal fluid (CSF), they are correlated with CSF measures in other CNS diseases [14]. To obtain platelet-poor plasma, blood samples were collected in EDTA-coated tubes (plasma) (BD Diagnostic 
Systems, NJ, USA), and were stored on ice. Plasma was separated by centrifugation at $40^{\circ} \mathrm{C}$ for $15 \mathrm{~min}$ at $1,500 \mathrm{x} \mathrm{g}$. This plasma was again re-centrifuged at $10,000 \mathrm{x} g$ and aliquots of poor platelet plasma were stored in polypropylene tubes at $-80 \mathrm{C}$ until assayed. BDNF levels were measured using a commercially available ELISA kit (RandD System), according to the manufacturer's instructions. However, during the standardization a sizable proportion of PLWH had BDNF values of $4000 \mathrm{pg} / \mathrm{mL}$ (ceiling effect), so the samples were 20 fold diluted. BDNF concentration in plasma was calculated based on a standard curve. The minimum detectable dose of BDNF is typically less than $62 \mathrm{pg} / \mathrm{mL}$. The repeatability of the BDNF ELISA, as measured by intra-assay precision, was $6 \%$, and the reproducibility as measured by inter-assay precision was $9 \%$. Coefficient of variation was $7.9(\mathrm{CV} \%=\mathrm{SD} /$ mean $\times 100 \%)$.

\section{Covariates}

Structured questionnaires were used to obtain sociodemographic information (i.e., age, income, marital status, education) medical history including mood disorders measured with the Beck depression inventory II [15] and treatments (antiretroviral and antidepressants). Participants were questioned regarding healthy behaviors such as type of diet and their level of physical activity (regular physical activity refers to leisure-time physical exercise). Other variables such as drug use (i.e., club drugs) were also considered in the model.

\section{Statistical Analyses}

Descriptive statistics (e.g. minimum, maximum, median and mean with standard deviation for each variable) and the frequency and percentage for each categorical variable were used to summarize the data, and to detect outliers and missing values. The normality of the distribution of primary outcomes of interest was examined with a normal probability plot. Natural log-transformed BDNF values were used in the analysis; however, to be comparable with prior publications, we are providing actual BDNF levels. Chi-square tests, t-tests or nonparametric tests were used for unadjusted analyses, as well as evaluation of potential confounders (where applicable).

Creating binary or categorical variables to measure the frequency of different types of risky sexual behaviors only covers a limited domain of sexual risk and lacks statistical power. To address this, we created a sexual-risk composite variable with an index that ranges from no sexual risk (a score of 0 ) to 10 . One point was added according to the degree of risk involved. For example, having unprotected vaginal sex contributed 1 point to the total score, while having sex during the menses or anal sex contributed 2 points. Having sex for money contributed 2 points, and number of partners contributed between 0 and 4 points, where $0=$ "no partner in the past 6 months" or "only had a monogamous relationship in the past 6 months". Two to five non-concurrent partners contributed 2 points, while multiple clients contributed up to 4 additional points if no protection was used.

General linear models were used to evaluate the independent association between high-risk behaviors (i.e. alcohol consumption, unprotected sex, sex for money) and BDNF. Variables adjusted for potential confounding effects included age, BMI, time of HIV diagnosis, and treatment. The validity of model assumptions was evaluated using analysis of residuals. P values less than 0.05 (2-tailed) were considered significant. Data analyses were performed using Statistical Package for the Social Sciences version 18.

\section{Results}

\section{Group characteristics}

The sample included both males (73\%) and females (27\%), ranging in age from 21 to 50 years ( $42 \pm 6$ years). Most participants were African-Americans (67\%) or Hispanics (25\%), with a smaller proportion of Caucasians (4\%) and Caribbeans (4\%). Among the 400 $\mathrm{PLWH}$, there was a wide range of BDNF levels in circulation, from 298 to $>20,000$ (mean $8384 \pm 6366 \mathrm{pg} / \mathrm{mL}$ ). BDNF levels differed by gender, with women exhibiting the highest levels $(9958.9 \pm 6578$ vs. $7470 \pm 6068 \mathrm{pg} / \mathrm{mL}, p=0.001$ ). Participants were dichotomized into two groups using the mean of the group as a cut-off point at $5000 \mathrm{pg} /$ mL: Group 1=low-BDNF ( $\leq 5000 \mathrm{pg} / \mathrm{mL}$ ), and Group 2=high-BDNF values $(>5000 \mathrm{pg} / \mathrm{mL})$. Please note that using a higher cutoff for women reached stronger $\mathrm{p}=$ values, but we did not uncover new relationships. Therefore, we decided to use a single cutoff point to simplify the presentation of the results.

Overall nutritional status, determined by levels of serum albumin, was within normal range for $99 \%$ of the participants $(4.3 \pm 0.4)$. No significant differences in nutritional status were noted between experimental cases and controls.

With regards to their clinical status, most had received a diagnosis of HIV infection more than a decade ago, and $74 \%$ had more than $200 \mathrm{CD} 4$ cell counts $(306 \pm 14$ cells $/ \mathrm{mL})$, and almost half exhibited undetectable viral loads. There were 28 participants who were not receiving antiretroviral therapy at baseline, but the distribution of treatment did not differ between the groups. As illustrated in Table 1, a sizable proportion of study participants were receiving treatment for depression. However, neither the depression status of the participants, nor the proportion of them receiving treatment was different between the groups.

\section{Alcohol and smoking}

Male hazardous alcohol users (HAU) were more likely to be smokers $(\mathrm{OR}=3.0,95 \% \mathrm{CI} 1.6-5.6, p=0.0001)$. Female HAU's were also twice as likely to smoke $(\mathrm{OR}=2.2,95 \% \mathrm{CI}$ : $1.1-4.8, p=0.013)$. Since evidence from animal studies suggests that nicotine may induce increased alcohol consumption, we examined this association. Smokers consumed alcohol more days in the week $(2.9 \pm 2.5$ vs. $1.8 \pm 0.4$ days, $p=0.001)$. Smokers also consumed more per occasion ( $4.9 \pm 0.33$ vs. 3.0 \pm 0.39 standard drinks, $p=.0001$ ) (Table 2).

\section{Alcohol and risk behaviors}

There were significant links between different risky behaviors. When we assessed specific associations between alcohol uses as a

\begin{tabular}{|c|c|c|c|}
\hline Variable & BDNF Group 1 & BDNF Group 2 & P Value \\
\hline Age & $43 \pm 5.7$ & $42 \pm 6.7$ & 0.7 \\
\hline Men & $75 \%$ & $70 \%$ & 0.1 \\
\hline Women & $25 \%$ & $30 \%$ & \\
\hline African American & $58 \%$ & $70 \%$ & \\
\hline Hiack Caribbean & $2 \%$ & $3 \%$ & \multirow{2}{*}{0.2} \\
\hline White & $28 \%$ & $23 \%$ & \\
\hline Education (years of school) & $11.6 \pm 2.0$ & $10.9 \pm 3.0$ & 0.6 \\
\hline Albumin g/dl & $4.0 \pm 0.6$ & $4.1 \pm 0.5$ & 0.9 \\
\hline Platelet Counts & $186 \pm 66$ & $241.8 \pm 139$ & 0.001 \\
\hline CD4 cell counts & $302 \pm 196$ & $391 \pm 248$ & 0.01 \\
\hline Viral Load Log & $3.1 \pm 1.2$ & $2.8 \pm 1.3$ & 0.02 \\
\hline Antiretroviral & $91 \%$ & $93 \%$ & 0.8 \\
\hline Beck Depression Score & $15.9 \pm 11.2$ & $16.4 \pm 12.7$ & 0.6 \\
\hline Antidepressants & $33 \%$ & $41 \%$ & 0.1 \\
\hline
\end{tabular}

Note: Demographic characteristics were expressed as percentages by BDNF group

Biological measures were presented as means and standard deviations

Table 1: Sociodemographic and clinical characteristics by BDNF groups. 
primary independent variable (categorized as non-HAU, HAU, heavy drinking) and several risky sex outcomes, HAU were more likely to be associated with a general risk-taking tendency. Indeed, when compared to non-HAU, HAU's scored higher in both sexual risk behaviors (3.1 \pm 1.9 vs. $2.6 \pm 1.8, p=0.001)$, and overall risk scores $(5.2 \pm 2.1$ vs. $3.2 \pm$ $1.9, p=0.01)$.

Male HAU were more likely to have sex with casual friends $(\mathrm{OR}=1.21,95 \% \mathrm{CI}: 1.0,1.4, p=0.01)$. HAU males were also more likely to always/frequently have sex under the influence of alcohol $(\mathrm{OR}=1.68$, 95\% CI: 1.0-2.9, $p=0.03$ ). HAU men were three times more likely not to use a condom when having sex with females $(\mathrm{OR}=3.1,95 \% \mathrm{CI}: 1.5$ $6.12, p=0.0005$ ), yet the same trend was not observed when having sex with males.

Female HAU were less likely to report that they never had sex under the influence of alcohol (OR=0.26, 95\% CI: $0.12-0.55, p=0.0001$ ). Of special concern, the majority of females (73\%) did not use condoms consistently during sexual intercourse.

\section{BDNF and addictions}

Notably, participants in the low-BDNF group were more likely to be hazardous drinkers (OR=1.6, 95\% CI: $1-2.4 ; p=0.01)$. Individuals in Group 1 drank more days ( $2.8 \pm 0.2$ vs. $2.3 \pm 0.2$ days/week, $p=0.04)$, and were also more likely to drink liquor than those in Group 2 (1.6 \pm 0.2 vs. $0.8 \pm 0.1$ drinks/occasion, $p=0.001$ ).

Compared to Group 2, Males in Group 1 were three times more likely to be smokers $(\mathrm{OR}=3.1,95 \% \mathrm{CI}$ : $1.6-5.6, p=0.0001)$ and a similar trend was observed for females $(\mathrm{OR}=2.2,95 \% \mathrm{CI}$ : 1.1-4.8, $p=0.013)$ (Table 3).

\section{Brain derived neurotrophic factor and high risk sexual behaviors}

Over one-third of the sample (35\%) reported either no sexual partners or only one. The remaining $65 \%$ had between two and one hundred partners in the past six months. Nearly half of the sample (48\%) reported exchanging sex for money. A significantly higher proportion of HIV infected women than men were reporting having sex for money ( $36 \%$ versus $67 \%, p=0.0001$ ).

Of concern, only $14 \%$ of them reported consistently using condoms, while $41 \%$ reported frequently being under the influence of drugs or alcohol during their sexual encounters. Despite years of education, high-risk sexual practices were common among this sample of PLWH, with $18 \%$ reporting unprotected sex during menses. Over half of the men (55\%) reported anal sex with women, and the remaining $18 \%$ with men.

We also assessed specific associations between BDNF as a primary independent variable and several risky sex practices. As shown in Figure 1 and in support of our main hypothesis, risky behaviors were more prevalent in the low BDNF group (Group 1). We found that Group 1 was 1.4 times more likely to report sex under the influence of drugs or alcohol than Group 2 (OR=1.4, 95\% CI: 0.9-2.26, $p=0.003$ ). Only $24 \%$ of the men and women reported regular condom use during the prior six months. Noteworthy, men in Group 1 tended to have multiple partners in the past 6 months (OR=2.1, 95\% CI: 1-6.1, $p=0.09$ ), but were less likely to use condoms during anal sex $(\mathrm{OR}=2.3,95 \% \mathrm{CI}$ : 1-6.9, $p=0.05)$ compared to Group 2. Men and women from Group 1 were three times as likely to report engaging in unprotected sex for money (OR=3.6; 95\% CI: 1-14.6, $p=0.03$ ).

\section{Risky behaviors and impulsivity}

Almost half of the study's population was hazardous alcohol users (48\%), with a median intake of $28 \pm 4$ alcoholic drinks weekly. Of particular concern, $37 \%$ of study participants scored above 8 on the AUDIT.

The alcohol beverage preference was similarly distributed: liquor was the most commonly consumed alcoholic beverage (45\%) and most liquor users $(60 \%)$ only drank liquor. One third of the

\begin{tabular}{|c|c|c|c|c|}
\hline \multirow{2}{*}{} & \multicolumn{5}{|c|}{ Coefficients $^{\text {a }}$} \\
\hline & Unstandardized Coefficients & $\begin{array}{c}\text { Standardized } \\
\text { Coefficients }\end{array}$ & \multirow{2}{*}{ Sig. } \\
\cline { 2 - 5 } & B & Std. Error & Beta \\
\hline BDNF & $-8.133 \mathrm{E}-007$ & 0.000 & -0.010 & -0.197 \\
\hline HAU & 0.115 & 0.055 & 0.104 & 2.083 \\
\hline Gender & -0.310 & 0.058 & -0.270 & -5.309 \\
\hline Antidepressants & 0.150 & 0.057 & 0.131 & 0.038 \\
\hline
\end{tabular}

a. Dependent Variable: SEXMONEY

Table 2: Multivariate analyses.

\begin{tabular}{|c|c|c|c|c|c|}
\hline \multirow{2}{*}{ Model } & \multicolumn{2}{|c|}{ Unstandardized Coefficients } & \multirow{2}{*}{$\begin{array}{c}\text { Standardized Coefficients } \\
\text { Beta }\end{array}$} & \multirow{2}{*}{$\mathbf{T}$} & \multirow{2}{*}{ Sig. } \\
\hline & B & Std. Error & & & \\
\hline Smoking & -1.388 & 0.249 & -0.288 & -5.577 & 0.000 \\
\hline Hazardous Alcohol Use & -1.658 & 0.230 & -0.365 & -7.196 & 0.000 \\
\hline BDNF & 6.976E-005 & 0.000 & 0.211 & 1.935 & 0.050 \\
\hline $\begin{array}{l}\text { BDNF } \times \text { Gender } \\
\text { interaction }\end{array}$ & $-4.713 E-005$ & 0.000 & -0.251 & -2.295 & 0.022 \\
\hline Antidepressants & -.690 & 0.228 & -0.151 & -3.029 & 0.003 \\
\hline Age & -.036 & 0.018 & -0.096 & -1.927 & 0.055 \\
\hline
\end{tabular}

Table 3: Multivariate analyses. 


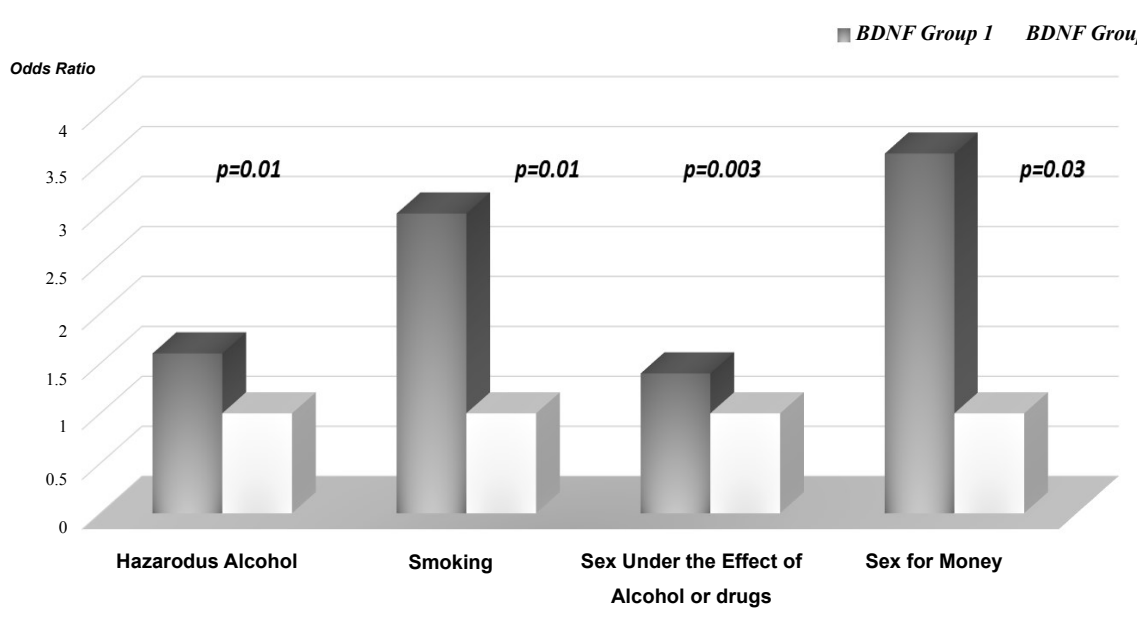

Figure 1: Sexual risks by BDNF groups.

participants consumed beer and $21 \%$ preferred wine. HAU and Non HAU were similar in their sociodemographic, behavioral and clinical characteristics.

On the Wisconsin Card Sorting Test-64 (WCST), when heavy drinkers were compared to those who drank less, groups differed significantly in the number of correct responses (62.17 \pm 16.5 vs. 67.0 $\pm 17.8, p=0.06)$. Heavy drinkers had more total errors ( $61.9 \pm 21.9$ vs. $54.39 \pm 24.19, p=0.03)$. They also had more perseverative errors $(49.04$ \pm 15.9 vs. $43.56 \pm 17.5, p=0.04$ ), but the failure-to-maintain- set variable was similar.

Although hazardous alcohol use was associated with poor performance on the WCST, WCST was less strongly associated with high-risk sexual behaviors. Only those reporting having sex most of time under the influence of drugs/alcohol had differences in the number of trials $(121.4 \pm 13.9$ vs. $126.3 \pm 7.1, p=0.05)$. Those reporting multiple partners also tended to have a higher percentage of errors 88.2 \pm 18 vs. $79.3 \pm 15.1 \%, p=0.08)$. No significant difference was observed between Groups 1 and 2 on any WCST scores in the multivariate analysis of covariance (MANCOVA) (Correct, $\mathrm{p}=0.82$; Perseverative Errors, $p=0.90$; Fail to maintain the set, $p=0.72$; Learning, $p=0.47$; Categories completed, $p=0.32$ ).

\section{Final analyses}

Multiple regression analysis was used to test if BDNF status significantly predicted participants' ratings of engaging in highrisk sexual behaviors. This was defined as two or more concomitant risks (i.e., unprotected anal or vaginal sex during menses, multiple partners, sex for money or having sex under the influence of drugs). BDNF ( $p=0.05)$, use of antidepressants $(p=0.003)$, hazardous alcohol use $(p=0.0001)$ and the BDNF $\times$ gender interaction all were significant predictors of high-risk sexual behaviors.

The multivariate analyses showed that three predictors explained $30 \%$ of the variance in engaging in sex for money. These variables were hazardous alcohol use $(\beta=.104, p=0.038)$, being female, and not taking antidepressants, $(\beta=-0.136, p=0.009)$. Neither age, income, education, impulsivity, nor BDNF remained in the model.

\section{Discussion}

Enhanced understanding of the factors associated with sexual risktaking, will be useful in designing new HIV prevention interventions.
Toward this end, our first inquiry was designed to answer the question, "to what extent can BDNF and serotonin status be directly related to sexual risk behaviors?" The current study demonstrated for the first time, that BDNF status was able to predict HIV risk behavior 6 months following initial assessment in PLWH. Risk taking behaviors in general have been associated with imbalances of neurochemicals in the brain including BDNF, dopamine and serotonin. Notably all these substances in addition to controlling cognition, also help with mood regulation [16]. This may explain why in our final models of sexual risk behaviors, both BDNF and the use of antidepressants were significant predictors. Given that depression was not directly related to risky behaviors, it could be proposed that mood stabilizers improve executive function and impulse control aside from treating mood disorders. This finding requires further investigation, but regardless of the mechanism treatment should be prioritized in this population.

While it is known that people living with HIV traditionally exhibit more than one risk behavior, researchers still analyze risk factors in isolation [16]. However, risk factors are more likely to occur in clusters. For example, research has been consistently showing that HIV infected populations across the globe are more likely to report higher use of alcohol and tobacco than the general population. The literature is also consistent in reporting that alcohol users more infrequently use condoms [17-19]. Yet the biological mechanism explaining these observations is largely unknown. In this regard, our data indicates that BDNF appears to play an important role in several of these risky behaviors. More specifically, low BDNF levels were related to hazardous alcohol use and smoking behaviors, suggesting that neurotransmitters are critical to the chain of risk-taking behaviors that are prevalent among people living with HIV. Results are not fully unexpected when ones consider that BDNF is highly expressed in brain regions controlling drug behaviors are also regulating other behavioral, sensory, cognitive, and emotional experiences [20]. This implies that dysregulation of key molecular signals, like BDNF and serotonin, could also influence other functions, such as sexual behaviors. These results may explain why HAU are less likely to use condoms and tend to engage in risky sexual practices. Findings are highlighting a unique opportunity for designing more global risk reduction strategies integrating biological principles and behavioral interventions.

For the same reason, our findings may aid in explaining why there is a link between smoking and HIV transmission [21-23]. First, an HIV infected smoker with altered BDNF levels will be more likely to 
engaging in other risky behaviors. Second, our group has previously shown that subjects with altered BDNF are more likely to have higher viral loads, and poorer immune status [24], and both may contribute to the explanation of these observations.

Of interest, we have found that alcohol behaviors are significantly related to BDNF levels. Altered BDNF levels can result from the direct effect of the virus on neurotrophic homeostasis, but based on models of addiction, it may also be the result of the drug-based alterations to the brain's neurotransmitter systems. Deficits in response inhibition were evident in the alcohol-hazardous group, yet impulsivity as measured by the Wisconsin Card-Sorting Task was not strongly associated with risky sexual behaviors or BDNF levels.

That being said, the limitations in the current study should be considered when interpreting the results. The study relied on selfreported assessments of sensitive behaviors and the frequencies of behavior. Even though the information was collected using computerized, reliable, and validated interviewing procedures, it should still be considered the lower-bound estimates of actual rates. In addition, the results are limited to those receiving care in South Florida, cautioning against generalizing the results to other settings. We have a large proportion of minorities, but since the inclusion of race/ethnicity did not modify the analyses, we feel very confident about the results that reflect more or less the socio demographics of the HIV epidemic in the USA. Finally, our original design does not include a healthy control population, as future studies need to be performed among the general population to confirm our findings. Acknowledging these limitations, we believe that our study findings support the immediate development of biological interventions based on BDNF, to attain global risk reduction interventions for PLWH.

In summary, our emerging data highlights the functional significance of BDNF status on both drug abuse (alcohol and smoking), as well as HIV transmission risk behaviors. Results suggest that highrisk taking individuals may have a biological makeup that may permit to identify such people so they can be targeted. Nonetheless, we suggest that further research be conducted to establish the validity of these findings in other populations.

\section{References}

1. CDC (2013) HIV Surveillance Report 25 .

2. Holtgrave DR, Hall HI, Wehrmeyer L, Maulsby C (2012) Costs, consequences and feasibility of strategies for achieving the goals of the National HIVIAIDS strategy in the United States: a closing window for success? AIDS and Behavior 16: $1365-1372$

3. Vermeulen-SE, Ten HM, Van LM, De Graaf R (2015) Clustering of health risk behaviours and the relationship with mental disorders. J Affect Disord 171: 111-119.

4. Hammond CJ, Mayes LC, Potenza MN (2014) Neurobiology of adolescent substance use and addictive behaviors: Prevention and treatment implications. Adolesc Med State Art Rev 25: 15-32.

5. Avdoshina V, Bachis A and Mocchetti I (2013) Synaptic dysfunction in human immunodeficiency virus type-1-positive subjects: Inflammation or impaired neuronal plasticity? J Intern Med 273: 454-465.

6. Míguez-Burbano M, Espinoza L, Cook RL, Vargas M, Bueno D, et al. (2013) Alcohol, brain derived neurotrophic factor and obesity among people living with HIV. J AIDS Clin Res 4: 245.
7. Maria Jose MB, Richardson E, Vargas M, Espinoza L, Lewis JE, et al. (2014) Mentholated cigarettes are related with abnormal brain-derived neurotrophic factor levels among smokers living with HIV. J Alcohol Drug Depend 2: 180.

8. Lorrain DS, Matuszewich L, Friedman RD, Hull EM (1997) Extracellular serotonin in the lateral hypothalamic area is increased during the post-ejaculatory interval and impairs copulation in male rats. J Neurosci 17: 9361-9366.

9. CDC (2014) HIV surveillance report 26.

10. Napper LE, Fisher DG, Reynolds GL, Johnson ME (2010) HIV risk behavior self-report reliability at different recall periods. AIDS Behav 14: 152-161.

11. National Institutes of Health (2015) Drinking levels defined.

12. Heatherton TF, Kozlowski LT, Frecker RC, Fagerstrom K (1991) The fagerstrom test for nicotine dependence: A revision of the fagerstrom tolerance questionnaire. $\mathrm{Br} \mathrm{J}$ Addict 86: 1119-1127.

13. Greve KW (2001) The WCST-64: A standardized short-form of the wisconsin card sorting test. Clin Neuropsychol 15: 228-34.

14. Radka SF, Holst PA, Fritsche M, Altar CA (1996) Presence of brain-derived neurotrophic factor in brain and human and rat but not mouse serum detected by a sensitive and specific immunoassay. Brain Research 709: 122-301.

15. Beck AT (1988) Psychometric properties of the beck depression inventory: Twenty-five years of evaluation. Clinical Psychology Review 8: 77-100.

16. Autry AE, Monteggia LM (2012) Brain-derived neurotrophic factor and neuropsychiatric disorders. Pharmacol Rev 64: 238-258.

17. Scott-Sheldon LAJ, Walstrom P, Carey KB, Johnson BT, Carey MP, et al. (2013) Alcohol use and sexual risk behaviors among individuals infected with HIV: A systematic review and meta-analysis 2012 to early 2013. Current HIV/ AIDS Rep10: 314-323.

18. Justice A, Sullivan L, Fiellin D; Veterans Aging Cohort Study Project Team (2010) HIVIAIDS, comorbidity, and alcohol: Can we make a difference? Alcohol Res Health 33: 258-266.

19. Leigh BC, Stall R (1993) Substance use and risky sexual behavior for exposure to HIV: Issues in methodology, interpretation and prevention. Am Psychol 48 1035-1045.

20. McEwen BS, Gianaros PJ (2010) Central role of the brain in stress and adaptation: Links to socioeconomic status, health and disease. Ann N Y Acad Sci 1186: 190-222.

21. Rahmanian S, Wewers ME, Koletar S, Reynolds N, Ferketich A, et al. (2011) Cigarette smoking in the HIV-infected population. Proc Am Thorac Soc 8: 313-319.

22. Marshall MM, McCormack MC, Kirk GD (2009) Effect of cigarette smoking on HIV acquisition, progression, and mortality. AIDS Educ Prev 21: 28-39.

23. Burns DN, Kramer A, Yellin F, Fuchs D, Wachter H, et al. (1991) Cigarette smoking: A modifier of human immunodeficiency virus type 1 infection? J Acquir Immune Defic Syndr 4: 76-83.

24. Míguez-Burbano MJ, Espinoza L, Bueno D, Vargas M, Trainor AB, et al. (2014) Beyond the brain: The role of brain-derived neurotrophic factor in viroimmune responses to antiretroviral therapy among people living with HIV with and without alcohol use. J Int Assoc Provid AIDS Care 13: 454-460. 